\title{
Panorama epidemiológico do processo de envelhecimento no mundo, Brasil e Piauí: evidências na literatura de 1987 a 2009
}

Recebido em: 20/06/2011

Aceito em: 26/07/2011

Selônia Patrícia Oliveira Sousa ${ }^{1}$ Sandra Beatriz Pedra Branca ${ }^{2}$

O objetivo deste estudo foi identificar e analisar as produções científicas nacionais sobre envelhecimento entre 1987 e 2009 . É uma revisão integrativa da literatura realizada por busca ativa na Biblioteca Virtual de Saúde (BVS). Foram identificadas e analisadas 14 produções. Após análise, emergiram duas categorias: dados demográficos e resultados em evidência. Constatou-se que o envelhecimento, por seu caráter multidimensional, faz com que o entendimento pelos profissionais seja tarefa obrigatória nas discussões, e ser idoso não significa necessariamente um aumento da qualidade de vida, principalmente nos países de terceiro mundo, devido ao processo das lentas políticas públicas de saúde.

Descritores: Envelhecimento, Saúde do Idoso, Transição Epidemiológica, Transição Demográfica.

\section{Epidemiological picture of the aging process in the world, and Piaui, Brazil: evidence from the literature from 1987 to 2009}

The objective of this study was to identify and analyze the national scientific production on aging from 1987 to 2009 . It is an integrative literature review, carried out by active search in the Virtual Health Library (VHL). It was identified and analyzed 14 productions. After the analysis emerged two categories: Demographic data and results in evidence. It was found that aging, its multidimensional nature, makes the understanding by professionals in the discussions is mandatory tasks, and being old does not necessarily mean an improved quality of life, especially in third world countries due to the slow process of political public health.

Descriptors: Aging, Health of the Elderly, Epidemiological Transition, Demographic Transition.

\section{Cuadro epidemiológico del proceso de envejecimiento en el mundo, Brasil y Piauí: evidencia de la literatura desde 1987 hasta 2009} El objetivo de este estudio fue identificar y analizar la producción científica nacional sobre el envejecimiento desde 1987 hasta 2009 . Se trata de una revisión de la literatura de integración, llevada a cabo por búsqueda activa en la Biblioteca Virtual en Salud (BVS). Se identificaron y analizaron 14 producciones. Tras el análisis se dividen en dos categorías: los datos demográficos y los resultados de las pruebas. Se encontró que el envejecimiento, su carácter multidimensional, hace que la comprensión por parte de los profesionales en las discusiones es tarea obligatoria, y ser viejo no significa necesariamente una mejor calidad de vida, especialmente en países del tercer mundo debido a la lentitud del proceso político la salud pública.

Descriptores: Envejecimiento, Salud del Anciano, Transición Epidemiológica, Transición Demográfica.

\section{INTRODUÇÃO}

A Organização Mundial de Saúde (OMS) definiu como idoso o indivíduo a partir de 65 anos nos países desenvolvidos, e a partir dos 60 anos para os países subdesenvolvidos. Considera-se ainda o processo de envelhecimento uma história de sucesso nas políticas de saúde públicas e sociais, constituindo uma das maiores vitórias da humanidade no último século. A queda de mortalidade e natalidade em países industrializados, iniciada no século passado, aconteceu em conjunto com a ampliação da cobertura vacinal, dos sistemas de proteção social e das melhorias das condições de habitação, alimentação, trabalho e saneamento básico ${ }^{(1,2)}$.

Esse fenômeno também faz parte da realidade da maioria das sociedades em desenvolvimento, como o Brasil. A partir de 1960, com o declínio da fecundidade nas Regiões Sul e Sudeste, iniciou-se o processo de envelhecimento populacional ${ }^{(3)}$.
Dados da própria OMS estimam que, para o ano de 2050, existirão cerca de 2 bilhões de pessoas com 60 anos ou mais no mundo, a maioria delas vivendo em países em desenvolvimento. Segundo as perspectivas epidemiológicas atuais, o Brasil deverá passar, entre 1960 e 2025, da 16a para a sexta posição mundial em termos de número absoluto de indivíduos com 60 anos ou mais, algo que está intrinsecamente relacionado às modificações sanitárias, sociais e políticas ${ }^{(3)}$. No Piauí, estado brasileiro localizado na Região Nordeste do país, a proporção de pessoas idosas está crescendo progressivamente, de forma rápida e intensa, passando de 7\%, em 1992, para 8,2\%, em 1999, destacando-se maior concentração na faixa etária entre 60 e 69 anos, com predominância do sexo feminino ${ }^{(4)}$.

Justifica-se este estudo, pois a busca de evidências tem sido muito usada na área da saúde e, mais recentemente, na de enfermagem, principalmente com o surgimento da enfermagem 
baseada em evidências e a revisão integrativa da literatura, que permite uma via de acesso ao conhecimento produzido.

O objetivo do presente estudo é identificar o perfil das produções e analisar as tendências dos resultados em evidência sobre o tema na produção científica nacional da enfermagem no período de 1987 a 2009.

\section{METODOLOGIA}

Estudo de natureza qualitativa-descritiva, desenvolvido por meio de uma revisão integrativa da literatura, recurso que proporciona a incorporação das evidências científicas na prática da saúde pública, tanto na pesquisa quanto na prática.

As etapas da elaboração da presente revisão integrativa foram: estabelecimento da hipótese e dos objetivos da revisão integrativa; estabelecimento de critérios de inclusão de artigos (seleção da amostra); definição das informações a serem extraídas dos artigos selecionados; análise dos resultados e discussão.

A questão norteadora desta revisão integrativa foi: quais as evidências teórico-metodológicas da literatura produzida sobre o envelhecimento no período de 1987 a 2009?

Para a seleção dos artigos, utilizou-se a base de dados da Biblioteca Virtual de Saúde (BVS). Os critérios de inclusão dos artigos definidos para a presente revisão integrativa foram: artigos publicados em português, com os textos completos disponíveis na base de dados selecionada, no período compreendido entre 1987 e 2009 . As palavras-chave utilizadas foram: "processo de envelhecimento; transição epidemiológica e demográfica; saúde do idoso, políticas públicas".

Após avaliação crítica dos resumos e artigos selecionados para verificar se respondiam plenamente à pergunta-guia, foram selecionadas e analisadas 14 produções, organizadas em dois eixos: dados demográficos e resultados em evidência.

\section{RESULTADOS}

\section{Eixo 1 - Dados demográficos}

"O envelhecimento populacional iniciou-se no fim do século 19 em alguns países da Europa Ocidental, espalhou-se pelo resto do primeiro mundo, no século passado, e se estendeu, nas últimas décadas, por vários países do terceiro mundo, inclusive o Brasil(5,6)."

$\mathrm{Na}$ América Latina, entre 1980 e 2000, ocorreu um aumento de $120 \%$ da população total (de 363,7 milhões para 803,6 milhões), enquanto o aumento da população acima de 60 anos será de $236 \%$ (de 23,3 milhões para 78,2 milhões), ou seja, duas vezes maior que o percentual de aumento da população em geral. Em longo prazo, as perspectivas são ainda mais impressionantes. Em alguns países, a população será de 16 milhões ou mais de pessoas acima de 60 anos em 2025, comparada às populações da mesma faixa etária em 1950. Entre os 11 países com as maiores populações de idosos daqui a 40 anos, oito situam-se na categoria de países em desenvolvimento, de acordo com os critérios atuais ${ }^{(5)}$.

No Brasil, a transição demográfica iniciada pelo declínio da mortalidade foi determinada mais pela ação médicosanitária do Estado do que pelas transformações estruturais.
Nas primeiras décadas do século 20, ela ocorreu graças às políticas urbanas de saúde pública, como vacinação, higiene pública e outras campanhas sanitárias, e, a partir da década de 1940, pela ampliação e pelo desenvolvimento tecnológico da atenção médica na rede pública ${ }^{(1)}$. Infelizmente, os tais ganhos não interferiram na desigualdade da distribuição de renda e de serviços.

Outra transformação ocorreu no Brasil em seu perfil epidemiológico, ou seja, em menos de 40 anos passamos de um índice de morbimortalidade típico de uma população jovem para um caracterizado por enfermidades crônicas, próprias das faixas etárias mais avançadas ${ }^{(7,8)}$.

Baseados nessa premissa, alguns autores afirmam que a mudança rápida no perfil epidemiológico, comparada ao perfil demográfico, propiciou grandes despesas com tratamentos médicos e hospitalares, ao mesmo tempo em que se configura um desafio para as autoridades sanitárias. O idoso consome mais serviços de saúde, as internações hospitalares são mais frequentes e o tempo de ocupação do leito é maior do que o de outras faixas etárias. Em geral, as doenças dos idosos são crônicas e múltiplas, perduram por vários anos e exigem acompanhamento médico e de equipes multidisciplinares permanentes e intervenções contínuas. Envelhecer sem nenhuma doença crônica é mais exceção do que regra ${ }^{(9)}$.

No Brasil, verificou-se um aumento de $258,3 \%$ das despesas com internações hospitalares pagas pelo SUS entre 1984 e 1991. O custo médio por internação elevou-se de US\$ 83,40 em 1984 para US\$ 268 em 1997. Tais políticas de saúde pública, desencadeadas no século 20 , resultaram no aumento da expectativa de vida da população brasileira, mas isso não significa dizer que houve melhoria da qualidade de vida dos idosos. O Brasil possui um contexto de desigualdades regionais e sociais, o que interfere na qualidade de vida ${ }^{(1,7)}$.

O perfil de morbimortalidade do Brasil está sendo alterado pelo envelhecimento populacional, fazendo com que o cenário caracterizado por uma população jovem, com maior incidência de doenças infecciosas, transforme-se em outro, típico de uma população mais envelhecida, em que predominam os agravos crônicos. Há uma correlação direta entre os processos de transição demográfica e epidemiológica. De modo geral, a queda inicial da mortalidade concentra-se seletivamente entre as doenças infecciosas e tende a beneficiar os grupos mais jovens da população. Esses "sobreviventes" passam a conviver com fatores de risco para doenças crônico-degenerativas. Sendo "preservadas" mais crianças, o efeito sobre a distribuição etária foi semelhante ao aumento da fecundidade, levando a um "rejuvenescimento" da população ${ }^{(1,10)}$.

Outros dados informam que há uma feminilização do envelhecimento no Brasil. Em 1950, as mulheres tinham maior esperança de vida ao nascer - cerca de sete anos e meio a mais $^{(11)}$. O número de mulheres idosas, confrontado com o de homens de mais de 60 anos de idade, já é superior e a proporção de idosas em relação à população total de mulheres supera aquela correspondente aos homens idosos. No Piauí, a estimativa do Censo Demográfico 2000 para a população total 
do município de Teresina correspondeu a 715.360 habitantes, dos quais 44.437 são pessoas com 60 anos ou mais ${ }^{(4)}$.

\section{Eixo 2 - Resultados em evidência}

A longevidade é, sem dúvida, um triunfo. Há, no entanto, diferenças profundas e importantes entre os países desenvolvidos e os em desenvolvimento. Enquanto nos primeiros o envelhecimento ocorreu associado às melhorias nas condições gerais de vida, nos outros, esse processo acontece de forma rápida, sem tempo para uma reorganização social e da área de saúde adequada para atender às novas demandas emergentes. Para o ano de 2050, a expectativa no Brasil, bem como em todo o mundo, é de que existirão mais idosos do que crianças abaixo de 15 anos ${ }^{(12)}$.

Sabe-se que envelhecer é um processo natural e fisiológico que se caracteriza pela diminuição progressiva da reserva funcional orgânica. Isso o torna, assim, mais suscetível a agravos e doenças, principalmente as de natureza crônicodegenerativas, que podem culminar na morte. Além dessas dificuldades, o idoso depara-se com problemas provocados pelos desequilíbrios sociais e regionais do desenvolvimento brasileiro, que acarretam carências nutricionais, sanitárias, educacionais, habitacionais. Ou seja, o envelhecimento não é homogêneo para todos os brasileiros, que sofrem influência dos processos de discriminação e exclusão associados ao gênero, à etnia, ao racismo, às condições sociais e econômicas, à região geográfica de origem e à localização de moradia ${ }^{(9)}$.

Outro ponto a ser lembrado é que o aumento biológico do percurso de vida da população tem ocorrido não por conquistas sociais, políticas, econômicas ou culturais, como se gostaria, mas sim pela difusão dos benefícios farmacêuticos, médicos e sanitários ${ }^{(7)}$. Os progressos da medicina têm conseguido prolongar a vida retangularizando a curva de mortalidade, e, por isso, cada vez mais, pessoas vivem até os 65 ou 70 anos.

Torna-se desejável que o Brasil tenha investimento efetivo em programas de suporte para idosos, como aposentadorias e pensões adequadas; oferta de serviços alternativos, como o centro-dia, o hospital-dia e outros; apoio em áreas de alimentação, transporte e assistência médica voltada aos problemas dos idosos. E também salários justos para os profissionais que cuidarão desses idosos; curso de reciclagem nessa área específica; implementação e implantação de ações interdisciplinares e transdisciplinares, entre outras. Porém, o mais importante é adequar os serviços de saúde ${ }^{(9)}$.

Pesquisas sobre o envelhecimento da população de Teresina-PI começaram a partir da experiência do Núcleo de Pesquisa e Estudo sobre a Terceira Idade (Nupeti), em 1995, na Universidade Federal do Piauí (UFPI). Esse fato gerou oficialmente, em agosto de 1998, por meio da resolução no 183/98, o Núcleo de Pesquisa e Extensão Universitária para a Terceira Idade (Nupeuti). ${ }^{(4)}$

\section{CONSIDERAÇÕES FINAIS}

Este trabalho permitiu-nos repensar sobre o idoso e seu envelhecimento, em diferentes âmbitos sociodemográficos, concluindo-se que, apesar de suas peculiaridades, a população idosa sofre o mesmo processo saúde-doença do restante, diferenciando apenas a intensidade das morbidades.

\section{Referências}

1. Chaimowicz FA. Saúde dos idosos brasileiros às vésperas do

século XXI: problemas, projeções e alternativas. Rev Saúde Pública.

1997;31(2):184-200.

2. Organização Mundial da Saúde. Envelhecimento ativo: uma política de

saúde. Brasília: Organização Pan-Americana da Saúde; 2005.

3. Ministério da Saúde (BR). Secretaria de Atenção à Saúde. Departamento

de Atenção Básica. Envelhecimento e saúde da pessoa idosa. Brasília:

Ministério da Saúde; 2006.

4. Alencar MSS, Carvalho CMRG. O envelhecimento pela ótica conceitual,

sociodemográfica e político-educacional: ênfase na experiência piauiense. Interface Comun Saúde Educ. 2009;13(29):435-44.

5. Kalache A, Veras RP, Ramos LR. O envelhecimento da população mundial: um desafio novo. Rev Saúde Pública. 1987;21(3):200-10.

6. Carvalho JAM, Garcia RA. O envelhecimento da população brasileira: um enfoque demográfico. Cad Saúde Pública. 2003;19(3):725-33.

7. Prochet TC, Ruiz TR, Correia I. Consideraçōes gerais sobre o

envelhecimento brasileiro. Rev Bras Enferm. 2006;5(3):168-73.

8. Pereira MG. Epidemiologia teoria e prática. $8^{\text {a }}$ ed. Rio de Janeiro:

Guanabara Koognan; 2005.

9. Santos SSC. Ensino da enfermagem gerontogeriatria e a complexidade.

Rev Esc Enferm USP. 2006;40(2):228-35.

10. Maia FOM, Duarte YAO, Lebrão ML. Análise dos Óbitos em idosos no

Estudo Sabe. Rev Esc Enferm USP. 2005;40(4):540-7.

11. Lei $n^{\circ} 1395$, de 10 de dezembro de 1999. Dispöe sobre a Política

Nacional de Saúde do Idoso [Internet]. [citado em 2009 Ago 29]. Disponível

em: http://www.planalto.gov.br/ccivil/LEIS/ 2003/L10.741.htm

12. Silva et al. Percepção de idosos de um centro de convivência sobre

envelhecimento. REME Rev Min Enferm. 2006;10(1):46-53. 